\section{Differential Recognition of the Outer and Inner Walls of Epidermal Cells by a Rust Fungus}

\author{
K. Mendgen \\ Fakultät für Biologie, Lehrstuhl Phytopathologie der Universität, \\ D-7750 Konstanz
}

Puccinia coronata Cda. f. sp. avenae Erikss., the oat crown rust fungus, differentiates highly specialized infection structures to penetrate into the leaf. At first the urediospore germ tube grows at right angles to the ridges of the cuticle until a stoma is encountered. There an appressorium with an infection peg is produced. The peg pushes the guard cells apart, penetrates into the substomatal chamber and forms the substomatal vesicle. From this vesicle, infection hyphae emerge (for explanation, see Fig. 1 a). When an infection hypha contacts a mesophyll cell, a haustorial mother cell is differentiated. Eventually a haustorium forms and pushes into a host cell. After establishment of the first haustorium, the infection hypha branches and more haustorial mother cells with secondary haustoria form.

A sequence of interactions between host and parasite appears to trigger the different phases of infection structure development [1]. It is the germ tube which recognises the surface structures of the cuticle, which in turn orients germ tube growth directionally [2]. The outer lips of the guard cells appear to induce appressorium formation [3], and they thus trigger the development of the remainder of the infection structure complex: the infection peg, substomatal vesicle and infection hypha. These latter structures can also be induced in the wheat stem rust fungus by heat treatment [4]. The induction of haustorial mother cells and haustoria has not previously been possible in vitro, although many rust fungi can be cultured artificially. This paper demonstrates that haustorium formation in a new type of artificial culture of oat crown rust is a result of a recognition between the walls of host and parasite.

To produce the different stimuli by the host, Avena sativa (cultivar Selma), to the germinating urediospores of the crown rust fungus (Puccinia coronata f. sp. avenae), an epidermal layer, stripped from oat coleoptiles [5], was spread on a microscope slide with either its outer (cuticular) wall surface up or its inner wall (mesophyll) surface up. The epidermal cell layer was floated either on sterile water or on a solution of $1 \%$ sucrose with $0.1 \%$ mineral fer-

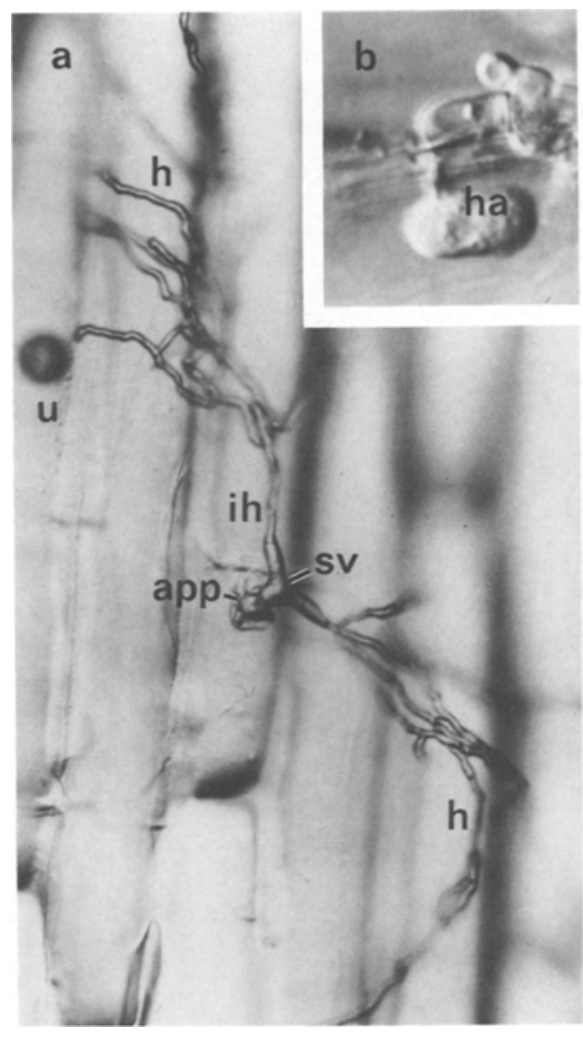

Fig. 1. a) Three-day-old rust mycelium with infection structures showing urediospore $(u)$ - the adhering germ tube has shriveled and is out of focus -, appressorium (app) with infection peg, substomatal vesicle (sv), and infection hypha $(i h)$. Many hyphae $(h)$ have formed haustorial mother cells with haustoria at their terminal ends. The latter cannot be seen in this upside view $(\times 200)$. b) Secondary haustorium ( $h a)$ with haustorial mother cell $(\times 600)$

tiliser (Wuxal from Schering A.G., WestBerlin, Germany). The inner or outer surfaces of the epidermal strips were inoculated with urediospores from a field collection of the crown rust fungus using a settling tower. Since these epidermals strips had no stomata in the area studied, the trig. gering stimulus normally provided by stomata was replaced by heat treatment $(4 \mathrm{~h}$ at $30^{\circ} \mathrm{C}$ ) beginning $2 \mathrm{~h}$ after inoculation. Fungal growth was studied by evaluating directional growth (=growth at right angles to the long axis of epidermal cells) of the germ tube, formation of infection structures, and formation of haustorial mother cells with haustoria at $20^{\circ} \mathrm{C}$. Two days after inoculation, directional growth of the urediospore germ tubes was much more pronounced on the surface of the outer side of the epidermal cells than on the inner surface (Fig. 2a). Appressoria were found only occasionally ( $2 \%$ ). Addi- 

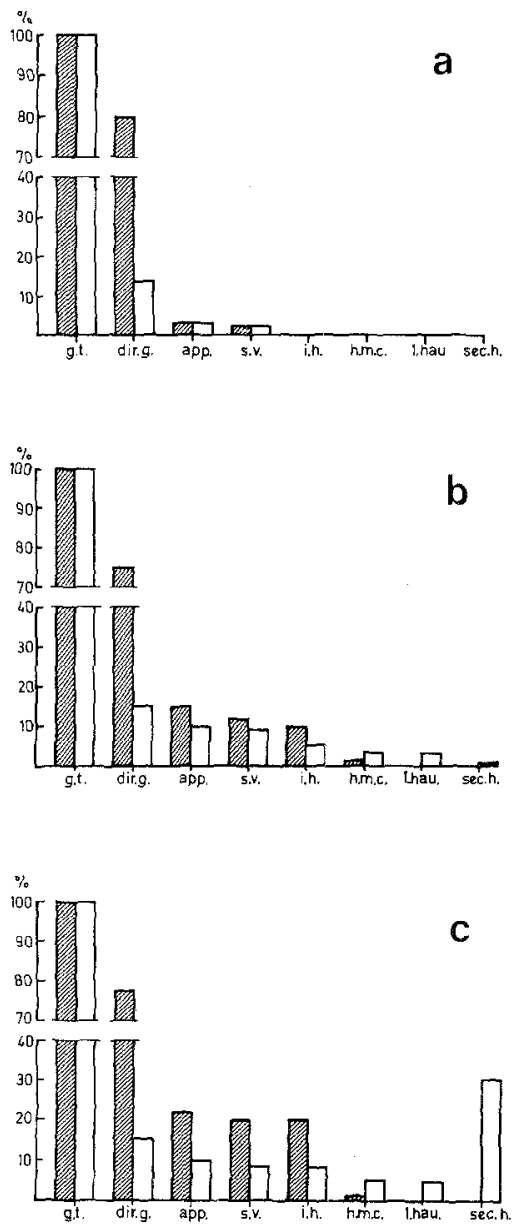

Fig. 2. a) Germ tubes showing directional growth (dir.g.), appressoria (app.), substomatal vesicles (s.u.), infection hyphae (i.h.), haustorial mother cells (h.m.c.), first haustoria (1. hau) and secondary haustoria (sec.h.) as a percentage of all urediospore germ tubes (g.t.) on the surface of the outer wall (清) and the inner wall ( $\square$ ) of an epidermal layer, two days after inoculation, 620 germ tubes counted. b) The different parts of infection structures, including haustoria, on the surface of the outer (四) and the inner ( $\square$ ) epidermal walls after a heat treatment $\left(4 \mathrm{~h}\right.$ at $30^{\circ} \mathrm{C}, 2 \mathrm{~h}$ after inoculation), six days after inoculation, 950 germ tubes counted. c) The different parts of infection structures, including haustoria (as above) after heat treatment (as above) and addition of a sucrose solution with mineral salts to the epidermal layer, six days after inoculation, 1200 germ tubes counted

tion of a solution with $1 \%$ sucrose and mineral salts to the epidermal strip did not alter the results (not shown). After a heat shock treatment (Fig. 2 b), about $10-20 \%$ of the germ tubes differentiated appressoria, infection pegs and substomatal vesicles on both sides of the epidermal cells. On the cuticular surface of the epidermis, haustorial mother cells formed rarely $(0.5 \%)$ and no haustoria were seen. On the surface of the inner wall, haustorial mother cells with haustoria were found regularly, and secondary haustoria were found occasionally ( $1 \%$ ). Addition of $1 \%$ sucrose and $0.1 \%$ mineral salts to the epidermal strip resulted in a dramatic increase in the number of secondary haustoria produced (Fig. $2 c$ ). About $4 \%$ of germinated spores produced mycelium with numerous haustorial mother cells and haustoria (Fig. 1 a, b).

Obviously, only infection hyphae form haustorial mother cells and haustoria after contact with the inner wall of epidermal cells, which corresponds to the walls of mesophyll cells. Attachment of the rust fungus to a mesophyll-type wall may be necessary to induce formation of haustorial mother cells and haustoria, as proposed for the bean rust fungus, Uromyces phaseoli [6].

The addition of simple nutrients such as sucrose and mineral salts (the latter can be omitted, which is not shown here) to the system greatly enhanced fungal growth and the formation of secondary haustoria. Five to ten days later, pustules with over
120 haustoria and urediospores with normal infectivity developed.

Supported by grant Me 523/9 from the Deutsche Forschungsgemeinschaft. I thank E. Dressler for excellent technical assistance and Dr. D.E. Harder, Dr. R.C. Staples and Dr. W.M. Masters for reading the manuscript.

Received June 8 and July 6, 1982

1. Staples, R., Macko, V.: Exp. Mycol. 4, 2 (1980); Wynn, W., Staples, R., in: Plant Disease Control, p. 45 (R. Staples, G. Toennissen, eds.). New York: Wiley 1981

2. Dickinson, S.: Ann. Bot. (London) N.S. 31, 89 (1949); Phytopathol. Z. 66, 38 (1969); 89, 97 (1977); Lewis, B., Day, J.: Trans. Br. mycol. Soc. 58, 139 (1972); Maheswari, R., Hildebrandt, A.: Nature 214, 1145 (1967)

3. Wynn, W.: Phytopathology 66, 136 (1976)

4. Dunkle, L., Allen, P.: ibid. 61, 649 (1971)

5. Bushnell, W., Dueck, J., Rowell, J.: Can. J. Bot. 45, 1719 (1967); Mendgen, K., Dressler, E.: Phytopathol. Z. (in preparation)

6. Mendgen, K.: Arch. Mícrobiol. 119, 113 (1978) 